\title{
CONOCIMIENTOS, ACTITUDES Y PRÁCTICAS SOBRE EL AUTOEXAMEN DE MAMA EN MUJERES DEL ÁREA RURAL DE CARTAGENA
}

\section{KNOWLEDGE, ATTITUDES AND PRACTICES ON THE BREAST SELF-EXAMINATION IN WOMEN OF THE RURAL AREA OF CARTAGENA}

\author{
Irma Castillo ${ }^{1}$, Cristina Bohórquez ${ }^{2}$, Jhoselys Palomino ${ }^{3}$, Laura Elles ${ }^{4}$, Lizeth Montero ${ }^{5}$
}

\begin{abstract}
${ }^{1}$ Enfermera. Magister en Salud Pública. Universidad de Cartagena, Facultad de Enfermería. Cartagena-Bolívar, Colombia, e-mail: icastilloa@unicartagena.edu.co; ${ }^{2}$ Enfermera. Universidad de Cartagena, Facultad de enfermería. Cartagena-Bolívar, Colombia: e-mail: cbohorquezm@unicartagena.edu.co; ${ }^{3}$ Enfermera. Universidad de Cartagena, Facultad de Enfermería. Cartagena-Bolívar, Colombia, e-mail: jpalominob1@unicartagena.edu.co; ${ }^{4}$ Enfermera. Universidad de Cartagena, Facultad de Enfermería. Cartagena-Bolívar, Colombia, e-mail: lauvane_29@hotmail.com; ${ }^{5}$ Enfermera. Universidad de Cartagena, Facultad de Enfermería. Cartagena-Bolívar, Colombia, e-mail: dulce-2505@hotmail.com
\end{abstract}

Rev. U.D.C.A Act. \& Div. Cient. 19(1):5-14, Enero-Junio, 2016

\section{RESUMEN}

El cáncer de mama se ha convertido en el segundo tipo de neoplasia más común en el mundo, por esta razón, la OMS promueve la lucha contra el cáncer, mediante el uso de la mamografía y el autoexamen de mama, especialmente, en las poblaciones vulnerables. El objetivo del estudio fue determinar los conocimientos, las actitudes y las prácticas sobre el autoexamen de mama, en mujeres del área rural de Cartagena. Se realizó un estudio descriptivo, con una muestra de 779 mujeres mayores de 20 años, residentes en el área rural, seleccionadas mediante muestreo por conglomerados, tomando como referencia los 15 corregimientos pertenecientes al distrito y realizando, posteriormente, afijación proporcional. Se aplicó una encuesta, que contempló las variables sociodemográficas, conocimientos, actitudes y prácticas sobre el autoexamen de mama. En la evaluación del nivel de información, percepciones y aplicación del autoexamen de mama, se encontró que el $73 \%$ de las mujeres conoce cómo se realiza el autoexamen; $77,8 \%$ están totalmente de acuerdo con que es bueno realizarlo; $49 \%$ nunca se lo ha realizado y solo tres mujeres tiene una práctica adecuada. Se concluyó que en la zona rural, a pesar que las mujeres han recibido información sobre la técnica del autoexamen, no se lo realizan de forma adecuada.

Palabras clave: Conocimientos, actitudes y práctica en salud, autoexamen de mamas, mujeres, salud de la mujer, medio rural.

\section{SUMMARY}

Breast cancer has become the second most common malignancy in the world type, for this reason the $\mathrm{WHO}$ is promoting the fight against cancer through the use of mammography and breast self-examination especially in vulnerable populations. The objective of the study was to determine the knowledge, attitudes and practices on breast self-examination in women in the rural area of Cartagena. He was a descriptive study with a sample of 779 women older than 20 years, residents in rural areas, selected by sampling by clusters, taking as a reference the 15 townships belonging to the district and subsequently doing proportional affixation. A survey which included Sociodemographic variables, knowledge, attitudes and practices on breast self-examination was applied. In the assessment of the level of information, insights and application of breast self-examination found that $73 \%$ of women know the self-diagnosis is performed, $77.8 \%$ are fully in agreement with that is good to do so, $49 \%$ have never done it and only three women have a good practice. It was concluded that in the rural area while the women have received information about the technique of self-diagnosis, these do not perform it is properly.

Key words: Health Knowledge, Attitudes, Practice, Breast SelfExamination, Women, Women's health, rural environment. 


\section{INTRODUCCIÓN}

Según la Agencia Internacional para la Investigación del Cáncer (IARC) y la Organización Mundial de la Salud (OMS), el cáncer de mama se ha convertido en el segundo tipo de neoplasia más común en el mundo y el más frecuente entre las mujeres (OMS, 2009; Arce et al. 2011).

En España, se diagnostican cada año entre 40 y 75 nuevos casos de este tumor maligno, por cada 100.000 mujeres y se producen alrededor de 6.000 fallecimientos anuales (Ferlay et al. 2013). México ocupa el primer lugar en incidencia del cáncer de mama en Latinoamérica y representa 1,34\% de todos los casos de cáncer, con un incremento global de, aproximadamente, 1,5\% anual (Arce et al. 2011).

Para Colombia, existe un aumento en las cifras del cáncer de mama; cada año mueren 2.649 mujeres por esta enfermedad, constituyéndose en la segunda neoplasia maligna más frecuente en mujeres del país, precedido por el cáncer de cuello uterino (Mendoza, 2013; Ministerio de Salud y Protección Social, 2014), con picos de incidencia más altos en ciudades como Barranquilla, Bogotá, Cartagena, Medellín y Cali (Ministerio de Salud y protección Social, 2014). En particular, para el caso de Cartagena, del total de las defunciones por cáncer registradas durante el 2013, la principal causa fue el cáncer de mama, que corresponde a un $18,2 \%$ y una tasa de mortalidad atribuible de 13,8 por 100.000 mujeres (DADIS, 2013).

Por la alta incidencia y la letalidad de esta enfermedad es importante aplicar programas que favorezcan la lucha contra el cáncer de mama, principalmente, en grupos de alto riesgo, con estrategias que promuevan el diagnóstico precoz y el tratamiento oportuno (Pardo, 2011).

Actualmente, existen métodos para la detección temprana del cáncer de mama, como son el autoexamen y la mamografía. A pesar de ser este último el medio de diagnóstico más utilizado, el autoexamen de mama (AEM) es un método usado como una importante estrategia de detección temprana, por ser una técnica de fácil realización, rápida y sin costos económicos para la mujer (Silva et al. 2013).

Este procedimiento no reduce la mortalidad por cáncer de mama, pero tener información sobre el autoexamen favorece el acceso a diagnósticos tempranos, principalmente, en países de bajos recursos económicos que no poseen infraestructura suficiente, para la realización rutinaria de mamografías (Gutiérrez Delgadillo et al. 2012).

La autoexploración mamaria, se recomienda en Colombia a partir de los 20 años de edad, por ser el primer recurso con que cuenta la mujer para llegar al diagnóstico temprano de algunos procesos patológicos de la glándula mamaria y, en especial, del cáncer y se refleja, en que más del $80 \%$ de los nódulos de mama son diagnosticados por la propia mujer (Ochoa et al. 2015).

De acuerdo con la Encuesta Nacional de Demografía y Salud (ENDS, 2010), en Colombia, el $90 \%$ de las mujeres mencionó saber que es el autoexamen de seno, conocimiento que es mayor en las zonas urbanas, con un $93,3 \%$, en mujeres con mayor nivel educativo y socioeconómico, a diferencia de la zona rural, donde solo es el $79 \%$. La mayor parte del conocimiento que tienen las mujeres sobre esta técnica resulta de los medios masivos de comunicación y de la instrucción recibida por los equipos básicos de salud en los primeros niveles de atención; lo que es semejante con otros estudios sobre el tema, realizados en poblaciones similares (Martínez et al. 2012).

Por ejemplo, solo el 34,6\% de mujeres peruanas de 20 a 49 años se realizaba el autoexamen de mama, práctica que es mayor entre mujeres de más de 30 años, de mayor índice de riqueza y que han sido examinadas por un profesional previamente (ENDS, 2010).

En cuanto a la práctica del autoexamen en Colombia, el $62 \%$ de las mujeres manifestó habérselo realizado, con una mayor frecuencia en las grandes ciudades, en mujeres de mayor nivel educativo, residentes en zonas urbanas y con un mayor nivel socioeconómico. Por departamentos, la mayor frecuencia de realización se reportó en Valle y, la menor, en Vaupés; la mayor proporción de mujeres que declararon haberlo realizado diariamente fueron mujeres del menor nivel educativo. En Arauca, el 55\% de las mujeres manifestaron hacerse el autoexamen cada mes; en San Andrés y Providencia, $71 \%$ de las mujeres se ha realizado alguna vez el autoexamen de mama, pero solo el $27,4 \%$ lo realiza mensualmente. Pese a ello, estas cifras no informan si la mujer tiene una técnica adecuada para la palpación o si inspecciona u observa los senos e incluye el pezón durante el examen realizado (ENDS,2010).

Estudiar los conocimientos, las actitudes y las prácticas sobre el autoexamen de mama es importante, no solo debido a las cifras de la enfermedad en el país, sino, además, porque la detección oportuna, mediante la autoexploración mamaria, es un procedimiento de mucho valor, que se refuerza desde el contacto que tiene la mujer con los servicios de salud, en especial, con los profesionales a cargo del cuidado que ayudan y orientan a la mujer durante la consulta, siendo este aspecto más significativo para las mujeres que están en zonas dispersas o zonas rurales, quienes tienen menor acceso a servicios e información en salud de calidad (Suasnabar \& Ramírez, 2011). 
Por todo lo anterior, esta investigación se propuso determinar los conocimientos, las actitudes y las prácticas sobre el autoexamen de mama, en las mujeres del área rural de Cartagena.

\section{MATERIALES Y MÉTODOS}

Se realizó un estudio descriptivo, con una población de referencia de 13.617 mujeres y un tamaño muestral estimado de 779 , probabilidad estimada del $62 \%$, nivel de confianza del $95 \%$ y un margen de error de $3,5 \%$; se incluyeron en el estudio, las mujeres de 20 a 49 años de edad, de acuerdo a lo establecido en la norma técnica, para la detección temprana del cáncer de mama y excluidas, las mujeres con patologías mamarias, que habitan en 15 corregimientos del área rural de Cartagena, seleccionadas mediante un muestreo por conglomerados, en cada uno de los corregimientos y posteriormente distribuidas por afijación proporcional, para su representatividad (Tabla 1).
Se empleó una encuesta sociodemográfica diseñada por los autores, con base en lo establecido en la Encuesta Nacional de Demografía y Salud de Colombia, para la caracterización de las mujeres encuestadas. Se aplicó, además, el instrumento propuesto por Sáenz \& Sánchez (2010), previa adaptación y validación por grupo de expertos, para medir los conocimientos, las actitudes y las prácticas sobre el autoexamen de mama en mujeres. Este instrumento auto administrado consta de 36 preguntas y los conocimientos fueron medidos mediante 8 ítems, con opciones de respuesta. Las actitudes fueron evaluadas mediante 14 enunciados, en donde las mujeres marcaron con una X si estaban totalmente de acuerdo (TA) (el máximo grado de conformidad con la afirmación); de acuerdo (A) (conformidad con la afirmación, pero no a un grado máximo); indeciso (I) (no saber si está en conformidad o en inconformidad con la afirmación); desacuerdo (DS) (no estar de acuerdo en lo absoluto con la afirmación) y las prácticas fueron medidas por medio de 14 preguntas, que presentaban opciones de respuesta abierta.

Tabla 1. Distribución por corregimientos de las mujeres del área rural de Cartagena. DANE (2010).

\begin{tabular}{|c|c|c|}
\hline CORREGIMIENTO & MUJERES MAYORES DE 20 AÑOS & $\begin{array}{c}\text { MUESTRA POR } \\
\text { CORREGIMIENTOS }\end{array}$ \\
\hline Arroyo Grande & 159 & 2 \\
\hline Arroyo De Las Canoas & 26 & 9 \\
\hline Arroyo De Piedra & 156 & 4 \\
\hline Punta Canoa & 76 & 16 \\
\hline Pontezuela & 276 & 16 \\
\hline Isla Fuerte & 280 & 182 \\
\hline La Boquilla & 3180 & 2 \\
\hline Islas Del Rosario & 36 & 68 \\
\hline Boca chica & 1194 & 23 \\
\hline Caño Del Oro & 409 & 26 \\
\hline Tierra Bomba & 452 & 135 \\
\hline Bayunca & 2364 & 187 \\
\hline Pasacaballos & 3268 & 61 \\
\hline Santana & 1059 & 39 \\
\hline Barú & 682 & $\mathbf{7 7 9}$ \\
\hline TOTAL & 13617 & 23 \\
\hline
\end{tabular}


La información recolectada fue incluida en una base de datos de Microsoft Excel y para el análisis estadístico, se utilizó el paquete estadístico SPSS 21.0. Se aplicó estadística descriptiva a los datos y se expresaron en frecuencias absolutas, frecuencias relativas, promedios y desviación estándar y medidas de tendencia central.

\section{Criterios éticos}

Este estudio, se fundamentó en la Resolución No. 008430 de 1993, del Ministerio de Salud, por medio de la cual se establecen las "Normas Científicas, Técnicas y Administrativas para la investigación en salud". De igual forma, se contó con el consentimiento informado por escrito del sujeto de investigación, en el cual, se explica los fines, la garantía de anonimato y la confidencialidad de los resultados obtenidos.

\section{RESULTADOS Y DISCUSIÓN}

En el estudio participaron 779 mujeres, de 20 a 49 años, pertenecientes al área rural de Cartagena, con una moda y promedio de edad de 36 y 33,6 años (DS 8,9), respectivamente. Con relación al estado civil predominó en un 54,2\% la unión libre, seguida por las solteras, con un $19 \%$ y, en último lugar, las separadas, con un $17,5 \%$. En cuanto a la ocupación, el $69,6 \%$ son amas de casa, seguidas de las trabajadoras independientes, con un 12,3\%. Realizaron bachillerato completo, $34,7 \%$ de las mujeres del estudio; llama la atención que el 13,9\% de ellas tienen un nivel de formación académica menor a la primaria completa y de éstas, el $4 \%$ no tienen estudios académicos, además viven en estrato socioeconómico bajo. Estos aspectos resultan relevantes, pues se ha observado mayor vulnerabilidad para el no uso de la información relacionada con el autocuidado de la salud, en el ámbito sexual y reproductivo, en aquellas mujeres que tienen una menor formación o instrucción académica y en las que no tienen parejas o relaciones estables; todo esto limita el acceso a la detección temprana de alteraciones y la búsqueda oportuna de atención profesional (Ospina et al. 2011) (Tabla 2).

Resultados similares, se obtuvieron en un estudio realizado en Tunja (Boyacá), donde se encontró que el promedio de edad de las participantes fue de 33,5 años, pertenecientes a los estratos socioeconómicos 1,2 y 3 y el 25,6\%, no terminó la educación básica (Ospina et al. 2011). Esta similitud evidencia la vulnerabilidad que presenta la población rural, por pertenecer a una zona con bajo nivel económico, educativo y con muchas necesidades básicas insatisfechas (Cruz \& Caballero, 2011).

Respecto a los conocimientos sobre el autoexamen de mama, un $73 \%$ conoce cómo se hace; un $67,7 \%$, afirma que todas las mujeres deben practicarlo y tan solo $22 \%$ de las
Tabla 2. Descripción de las variables socio demográficas de las mujeres entre 29 y 49 años, del área rural de Cartagena.

\begin{tabular}{|c|c|c|}
\hline Estado Civil & $\begin{array}{l}\text { Frecuencia } \\
\text { absoluta }\end{array}$ & $\begin{array}{c}\text { Frecuencia } \\
\text { relativa }\end{array}$ \\
\hline Separada & 136 & 17,5 \\
\hline Soltera & 148 & 19,0 \\
\hline Unión libre & 422 & 54,2 \\
\hline Casada & 59 & 7,6 \\
\hline Viuda & 14 & 1,8 \\
\hline Ocupación & $\begin{array}{c}\text { Frecuencia } \\
\text { absoluta }\end{array}$ & $\begin{array}{l}\text { Frecuencia } \\
\text { relativa }\end{array}$ \\
\hline Ama de casa & 542 & 69,6 \\
\hline Desempleada & 18 & 2,3 \\
\hline Estudiante & 75 & 9,6 \\
\hline Empleada & 48 & 6,2 \\
\hline Independiente & 96 & 12,3 \\
\hline Nivel educativo & $\begin{array}{c}\text { Frecuencia } \\
\text { absoluta }\end{array}$ & $\begin{array}{c}\text { Frecuencia } \\
\text { relativa }\end{array}$ \\
\hline Sin estudios & 31 & 4,0 \\
\hline Primaria incompleta & 77 & 9,9 \\
\hline Primaria completa & 78 & 10,0 \\
\hline $\begin{array}{l}\text { Bachillerato } \\
\text { incompleto }\end{array}$ & 187 & 24,0 \\
\hline $\begin{array}{l}\text { Bachillerato } \\
\text { completo }\end{array}$ & 270 & 34,7 \\
\hline Técnico & 89 & 11.4 \\
\hline Tecnólogo & 17 & 2,2 \\
\hline Universitario & 30 & 3,9 \\
\hline $\begin{array}{c}\text { Estrato } \\
\text { socioeconómico }\end{array}$ & $\begin{array}{c}\text { Frecuencia } \\
\text { absoluta }\end{array}$ & $\begin{array}{l}\text { Frecuencia } \\
\text { relativa }\end{array}$ \\
\hline 1 & 756 & 97,0 \\
\hline 2 & 22 & 2,8 \\
\hline 3 & 1 & 0,1 \\
\hline
\end{tabular}

encuestadas, entiende que se hace a partir de los 20 años. Un $51,1 \%$ opina que al practicar esta técnica, se asegura un buen estado de salud; para el 30,8\%, el autoexamen se debe realizar una vez al mes y para el $32,2 \%$, diariamente. El $57,1 \%$ manifestó que el beneficio de practicarlo es detectar el cáncer tempranamente y curarlo (Tabla 3). Estos resultados difieren a los registrados en mujeres de Cartagena, donde solo el 33,8\% conoce la técnica, mientras que las que no la conocen o no responden, equivale al $67,2 \%$ de la muestra. El $61,2 \%$ de las mujeres de este estudio manifiestan que el momento adecuado para comenzar a realizarse el autoexamen de mama es la 
adolescencia (Toro et al. 2014). Cuando estos resultados son analizados a la luz de la Resolución 0412 de 2000, que establece la norma técnica para la detección temprana del cáncer de seno, este procedimiento se debe efectuar a partir de los 20 años, con el fin de ayudar a la mujer a familiarizarse con la forma en la que deberían lucir la apariencia de sus senos y con la sensibilidad y la textura que deberían tener; además, debería permitirle detectar y realizar un seguimiento de cualquier clase de cambio que pudiera surgir repentinamente, en alguno de ellos (Ministerio de Salud, 2000).

Asimismo, en un estudio realizado en Lima (Perú), se reporta que el $62,5 \%$ de las mujeres desconoce la frecuencia en la que se debe realizar el autoexamen y el $78,8 \%$, ignora su utilidad, para la detección temprana del cáncer (Sáenz \& Sánchez, 2010). El conocimiento es la base informativa que da significado a las creencias y prácticas frente a una situación. Cuando la información es completa y clara el resultado obtenido debe ser el adecuado, porque se ve enmarcado en la actitud y puede ser mediado por la motivación y las habilidades personales; se esperaría que un mejor conocimiento sobre el autoexamen generará entre las mujeres una práctica adecuada y regular del mismo (Ochoa et al. 2015).

Un aspecto importante de este estudio es que la fuente que ofrece mayor información sobre el autoexamen de mama a las mujeres es el centro de salud. Esto es similar a lo encontrado en un estudio realizado en Lima (Perú), donde el 31,5\% de las encuestadas manifiestan que la fuente más importante es el profesional de la salud; sin embargo, los resultados difieren de los encontrados por Bell \& Oduardo (2015), en mujeres de una comunidad venezolana, donde el $86 \%$ de la población recibía información acerca del autoexamen de mama mediante la promoción gráfica o familiares, los amigos y un grupo menor, por el personal de salud. Esto se convierte en una oportunidad para los profesionales de la salud, en especial, el profesional de enfermería, quien trabaja en el área rural, encargado de coordinar los servicios de salud que se brindan en los diferentes corregimientos; por tanto, juega un papel importante como educador, brindando información a las mujeres sobre este examen, de forma clara y de buena calidad, para garantizar un conocimiento completo, que se refleje en una mejor práctica de este procedimiento (Bell \& Oduardo, 2015).

Las mujeres de 30 a 49 años de edad fueron quienes presentaron un mayor porcentaje de conocimiento sobre la técnica de autoexamen de mama (Tabla 4), resultados similares a los registrados en un estudio realizado en Cuba, donde las mujeres de 40 años o más, fueron quienes refirieron conocer esta técnica (Martínez et al. 2012); también son similares a los obtenidos en Brasil, donde las mujeres mayores de 40 años mostraron mejores conocimientos acerca del autoexamen de mama (Silva et al. 2013). Lo anterior, se debe a que a medida que avanza el ciclo vital individual aumentan las regularidades en los estilos de vida y así quedó evidenciado en un estudio realizado en Chile, donde las personas con promedios de edad mayor presentaban más responsabilidad con su salud (Vidal et al. 2014).

Tabla 3. Conocimientos de las mujeres entre 20 y 49 años, del área rural de Cartagena.

\begin{tabular}{|c|c|c|}
\hline $\begin{array}{l}\text { ¿Conoce cómo se realiza el } \\
\text { autoexamen de mama? }\end{array}$ & $\begin{array}{l}\text { Frecuencia } \\
\text { absoluta }\end{array}$ & $\begin{array}{c}\text { Frecuencia } \\
\text { relativa }\end{array}$ \\
\hline $\mathrm{Si}$ & 569 & 73.0 \\
\hline No & 210 & 27.0 \\
\hline $\begin{array}{l}\text { ¿Quiénes se deben realizar el } \\
\text { auto examen de mama? }\end{array}$ & $\begin{array}{l}\text { Frecuencia } \\
\text { absoluta }\end{array}$ & $\begin{array}{l}\text { Frecuencia } \\
\text { relativa }\end{array}$ \\
\hline Mujeres con vida sexual activa & 35 & 4,5 \\
\hline No sabe & 46 & 5,9 \\
\hline $\begin{array}{l}\text { Todas las mujeres a partir de los } \\
20 \text { años }\end{array}$ & 144 & 22,0 \\
\hline Todas las mujeres & 527 & 67,7 \\
\hline $\begin{array}{l}\text { ¿Para qué se realiza el } \\
\text { autoexamen de mama? }\end{array}$ & $\begin{array}{c}\text { Frecuencia } \\
\text { absoluta }\end{array}$ & $\begin{array}{c}\text { Frecuencia } \\
\text { relativa }\end{array}$ \\
\hline Para buscar alguna enfermedad & 113 & 14,5 \\
\hline Para detectar cáncer & 266 & 34,1 \\
\hline Para estar segura de estar sanas & 398 & 51,1 \\
\hline $\begin{array}{l}\text { ¿En qué momento se debe } \\
\text { realizar el autoexamen de mama? }\end{array}$ & $\begin{array}{c}\text { Frecuencia } \\
\text { absoluta }\end{array}$ & $\begin{array}{c}\text { Frecuencia } \\
\text { relativa }\end{array}$ \\
\hline Una vez al año & 140 & 18,0 \\
\hline Una vez al mes & 240 & 30,8 \\
\hline $\begin{array}{l}4 \text { a } 10 \text { días desde el primer día } \\
\text { de la menstruación }\end{array}$ & 39 & 5,0 \\
\hline $\begin{array}{l}8 \text { días después de la } \\
\text { menstruación }\end{array}$ & 94 & 12,1 \\
\hline Diario & 251 & 32,2 \\
\hline No sabe & 12 & 1,5 \\
\hline Cualquier día & 1 & 0,1 \\
\hline $\begin{array}{l}\text { ¿De dónde obtuvo información } \\
\text { para realizar el autoexamen? }\end{array}$ & $\begin{array}{c}\text { Frecuencia } \\
\text { absoluta }\end{array}$ & $\begin{array}{l}\text { Frecuencia } \\
\text { relativa }\end{array}$ \\
\hline Centro de salud & 379 & 48,6 \\
\hline Familiares & 44 & 5,6 \\
\hline Libros y folletos & 24 & 3,1 \\
\hline Televisión & 111 & 14,2 \\
\hline Radio & 7 & 0,9 \\
\hline Internet & 3 & 0,4 \\
\hline Ninguno & 45 & 5,8 \\
\hline Todos & 166 & 20,2 \\
\hline
\end{tabular}


Con relación a las actitudes de las encuestadas hacia el autoexamen de mama, el 77,8\% están totalmente de acuerdo con las bondades o beneficios de realizarse el autoexamen; $68,3 \%$ consideran que es importante realizarlo; el $63,7 \%$ lo practican para protegerse de enfermedades; $64,3 \%$ tiene tiempo para realizarse el autoexamen y no les da pereza y al 55,7\%, no se le olvida practicarlo (Tabla 5). Las actitudes positivas encontradas en este estudio son similares a las obtenidas por (Ochoa et al. 2015), en su estudio adelantado en mujeres del área rural de Cuenca, donde más del $90 \%$ de ellas estuvieron de acuerdo con la realización del autoexamen de mama y consideran que es de fácil realización. Esto difiere con los resultados encontrados por Sáenz \& Sánchez (2010), en una investigación realizada en Lima (Perú), donde menos de la mitad de las encuestadas estaban totalmente de acuerdo con los beneficios de realizarse el autoexamen de mama y se sentían indecisas sobre si practicarse el autoexamen las protege de enfermedades. También afirman que no se lo realizan por falta de tiempo, se les olvida y no creen que sea importante llevar a cabo esta técnica.

Tener una actitud positiva, se convierte en un mediador para tener una buena práctica en este procedimiento, porque favorece que la mujer acuda tempranamente al sistema de salud cuando detecte alguna alteración, pueda acceder a un diagnóstico más oportuno y obtenga los cuidados y el tratamiento que necesita (Ochoa et al. 2015).

No es suficiente el conocimiento y la buena actitud que poseen las mujeres hacia el autoexamen de mama para una buena práctica, pues manifiestan conocer la técnica y tener una actitud positiva, pero solo el $49 \%$ se ha realizado anteriormente el autoexamen y, de este porcentaje, solo el $54,2 \%$ lo realiza cada mes; el 34,7\%, una vez al mes después de la menstruación y solo el 5,3\%, lo practica del cuarto al décimo día desde el primer día de la menstruación (Tabla 6). Estos resultados difieren con lo consignado en la Norma técnica para la detección temprana del cáncer de seno en Colombia, donde se establece que este procedimiento se debe realizar mensualmente, del 4 a 10 día desde del primer día de la menstruación y si la mujer ya no menstrúa se debe elegir un día al mes y realizarlo, pero son similares a los resultados encontrados por Silva et al. (2013), donde, a pesar que el $86,4 \%$ de las mujeres manifestaron conocer la técnica y practicarla, no la efectúan con la periodicidad adecuada.

Cuando la práctica de autoexamen de mama se realiza en otros momentos o con una frecuencia distinta, se corre el riesgo de que la mujer se examine sus senos en un momento del ciclo menstrual, en el cual, las mamas se encuentren inflamadas y dolorosas, llevándolas a encontrar alteraciones propias de las acciones hormonales y que no se derivan de alguna afección (Fernández et al. 2012).

En cuanto a la técnica para realizar del autoexamen, el $82,7 \%$, se observa y $55,8 \%$, se palpa; estos resultados son similares a los obtenidos por Cervantes et al. (2011), quienes hallaron que el $25 \%$ de las encuestadas se miran frente al espejo y se palpan; este aspecto es muy importante, porque el autoexamen no es solo palparse los senos, en forma

Tabla 4. Distribución de conocimientos sobre el autoexamen de mama, según grupo etéreo.

\begin{tabular}{|c|c|c|c|c|c|c|c|c|}
\hline \multicolumn{9}{|c|}{ ¿Conoce cómo se realiza el autoexamen de mama? } \\
\hline Edad & \multicolumn{4}{|c|}{ NO } & \multicolumn{4}{|c|}{ SI } \\
\hline 20 a 29 años & \multicolumn{2}{|c|}{108} & \multicolumn{2}{|c|}{$38,3 \%$} & \multicolumn{2}{|c|}{174} & \multicolumn{2}{|c|}{$61,7 \%$} \\
\hline 30 a 39 años & \multicolumn{2}{|c|}{60} & \multicolumn{2}{|c|}{$20,2 \%$} & \multicolumn{2}{|c|}{237} & \multicolumn{2}{|c|}{$79,8 \%$} \\
\hline 40 a 49 años & \multicolumn{2}{|c|}{42} & \multicolumn{2}{|c|}{$21,0 \%$} & \multicolumn{2}{|c|}{158} & \multicolumn{2}{|c|}{$79,0 \%$} \\
\hline \multicolumn{9}{|c|}{ ¿Quiénes se deben realizar el auto examen de mama? } \\
\hline Edad & \multicolumn{2}{|c|}{$\begin{array}{l}\text { Mujeres con vida } \\
\text { sexual activa }\end{array}$} & \multicolumn{2}{|c|}{ Todas la mujeres } & \multicolumn{2}{|c|}{$\begin{array}{c}\text { Toda las mujeres a partir } \\
\text { de los } 20 \text { años }\end{array}$} & \multicolumn{2}{|c|}{ No sabe } \\
\hline 20 a 29 años & 20 & $7,1 \%$ & 178 & $63,1 \%$ & 61 & $21,6 \%$ & 23 & $8,2 \%$ \\
\hline 30 a 39 años & 9 & $3,0 \%$ & 206 & $69,4 \%$ & 68 & $22,9 \%$ & 14 & $4,7 \%$ \\
\hline 40 a 49 años & 6 & $3,0 \%$ & 143 & $71,5 \%$ & 42 & $21,0 \%$ & 9 & $4,5 \%$ \\
\hline \multicolumn{9}{|c|}{ ¿Para qué se realiza el autoexamen de mama? } \\
\hline Edad & \multicolumn{2}{|c|}{$\begin{array}{l}\text { Buscar alguna en- } \\
\text { fermedad }\end{array}$} & \multicolumn{2}{|c|}{ Detectar cáncer } & \multicolumn{2}{|c|}{$\begin{array}{c}\text { Estar segura de estar } \\
\text { sanas }\end{array}$} & \multicolumn{2}{|c|}{ No sabe } \\
\hline 20 a 29 años & 40 & $14,2 \%$ & 90 & $31,9 \%$ & 151 & $53,5 \%$ & 1 & $0,4 \%$ \\
\hline 30 a 39 años & 42 & $14,1 \%$ & 97 & $32,7 \%$ & 157 & $52,9 \%$ & 1 & $0,3 \%$ \\
\hline 40 a 49 años & 31 & $15,5 \%$ & 79 & $39,5 \%$ & 90 & $45,0 \%$ & 0 & $0,0 \%$ \\
\hline
\end{tabular}


Tabla 5. Actitudes de las mujeres entre 20 y 49 años, del área rural de Cartagena.

\begin{tabular}{|l|c|c|c|c|c|c|c|c|}
\hline \multicolumn{1}{|c|}{ Proposiciones } & TA & \% & A & \% & I & \% & DS & $\%$ \\
\hline Es bueno realizarlo & 606 & 77,8 & 170 & 21,8 & 2 & 0,3 & 1 & 0,1 \\
\hline $\begin{array}{l}\text { Yo me realizo el autoexamen de mama } \\
\text { para protegerme de enfermedades }\end{array}$ & 496 & 63,7 & 200 & 25,7 & 11 & 1,4 & 72 & 9,2 \\
\hline $\begin{array}{l}\text { El autoexamen de mama me } \\
\text { da muchos beneficios }\end{array}$ & 531 & 68,2 & 202 & 25,9 & 13 & 1,7 & 33 & 4,2 \\
\hline $\begin{array}{l}\text { El personal de salud influye en la } \\
\text { realización del examen }\end{array}$ & 452 & 58,0 & 256 & 32,9 & 19 & 2,4 & 52 & 6,7 \\
\hline $\begin{array}{l}\text { Me preocupa la aparición de } \\
\text { bultos u otros }\end{array}$ & 531 & 68,2 & 202 & 25,9 & 13 & 1,7 & 33 & 4,2 \\
\hline Es un pecado realizarlo & 30 & 3,9 & 20 & 2,6 & 26 & 3,3 & 703 & 90,2 \\
\hline $\begin{array}{l}\text { La religión influye en la decisión de } \\
\text { realizar el examen }\end{array}$ & 38 & 4,9 & 43 & 5,5 & 22 & 2,8 & 676 & 86,8 \\
\hline $\begin{array}{l}\text { El examen me ayuda a detectar } \\
\text { enfermedades malignas y benignas }\end{array}$ & 367 & 47,1 & 265 & 34,0 & 50 & 6,4 & 97 & 12,5 \\
\hline No tengo tiempo para realizarlo & 37 & 4,7 & 145 & 18,6 & 96 & 12,3 & 501 & 64,3 \\
\hline Me da pereza & 37 & 4,7 & 145 & 18,6 & 96 & 12,3 & 501 & 64,3 \\
\hline Se me olvida & 41 & 5,3 & 198 & 25,4 & 106 & 13,6 & 434 & 55,7 \\
\hline No creo que sea importante & 27 & 3,5 & 66 & 8,5 & 82 & 10,5 & 604 & 77,5 \\
\hline $\begin{array}{l}\text { El personal de salud no me ha } \\
\text { explicado sobre esto }\end{array}$ & 59 & 7,6 & 98 & 12,6 & 90 & 11,6 & 532 & 68,3 \\
\hline
\end{tabular}

Totalmente de acuerdo (TA); de acuerdo (A); indeciso (I); desacuerdo (DS).

sistemática en busca de bultos, sino que se debe hacer una inspección frente a un espejo, buscando deformidades, retracción o anomalías del pezón y cambios de coloración en la piel (Yépez et al. 2012).

Respecto a la forma de realizar el procedimiento, el $83,8 \%$, se palpa con la yema de los dedos; $87,7 \%$, se autoexaminan un seno por vez y $80,6 \%$, incluye el pezón, cuando se lo practica. Solo tres mujeres tienen una práctica adecuada del autoexamen, pues cumplen con la técnica adecuadamente, además de realizarlo con periodicidad mensual y en los días del ciclo menstrual recomendados. Estos datos son superiores a los obtenidos en Cartagena, donde reportan que el $42 \%$ autoexaminan un seno a la vez; el $43,3 \%$, se palpa con la yema de los dedos y el $31,8 \%$, incluye el pezón en el momento que lo realizan (Toro et al. 2014). Una buena práctica cumple con las variables requeridas, para que el autoexamen de mama sea completo y útil, permitiendo a la mujer conocer el aspecto normal de sus senos y detectar más fácilmente cualquier síntoma o signo de alarma, en el caso de que se presente algún cambio y que pueda ser reportado o comentado a su médico tempranamente (Suasnabar \& Ramírez, 2011).
En general, aunque las mujeres reportan tener conocimientos sobre el autoexamen de mama y una buena actitud frente a la importancia de este método en el cuidado de la salud, no son completos y suficientes para impactar positivamente en la práctica, mostrándose un conocimiento ambiguo sobre la técnica y generándose una barrera para la realización del autoexamen, peor aún, una mala práctica, que genera resultados erróneos o confusos para la mujer (Ochoa et al. 2015).

Los resultados encontrados permiten concluir que los conocimientos que tienen las mujeres del área rural de Cartagena sobre el autoexamen de mama son bajos, presentan una actitud favorable hacia este procedimiento, pero la práctica es inadecuada. La mayoría de las mujeres dice conocer cómo se realiza el autoexamen de mama, pero desconocen la frecuencia y el tiempo del ciclo menstrual, en el cual, se debe realizar, lo que puede llevar a que se encuentren alteraciones derivadas de las acciones hormonales o que se ignoren problemas potencialmente perjudiciales para la mujer.

Se recomienda para próximas investigaciones indagar en las entrevistas sobre factores de riesgo, antecedentes familiares 
Tabla 6. Práctica de las mujeres entre 20 y 49 años, del área rural de Cartagena.

\begin{tabular}{|c|c|c|}
\hline ¿Se ha realizado anteriormente el autoexamen de mama? & Frecuencia absoluta & Frecuencia relativa \\
\hline SI & 382 & 49,0 \\
\hline ¿Se realiza el autoexamen cada mes? & Frecuencia absoluta & Frecuencia relativa \\
\hline SI & 208 & 54,2 \\
\hline ¿En qué días se realiza el autoexamen? & Frecuencia absoluta & Frecuencia relativa \\
\hline $1 \mathrm{vez}$ al año & 8 & 2,1 \\
\hline 4 a 10 desde el primer día de la menstruación. & 20 & 5,3 \\
\hline Diario & 141 & 37,1 \\
\hline ¿Cada cuánto se realiza el autoexamen? & Frecuencia absoluta & Frecuencia relativa \\
\hline Anual & 21 & 5,5 \\
\hline Diario & 124 & 32,6 \\
\hline Mensual & 168 & 44,2 \\
\hline ¿Qué técnica utiliza? & Frecuencia absoluta & Frecuencia relativa \\
\hline Observación & 8 & 2,1 \\
\hline Palpación & 58 & 15,3 \\
\hline Ambas & 316 & 82,7 \\
\hline ¿En qué posición observa sus senos? & Frecuencia absoluta & Frecuencia relativa \\
\hline Acostada & 147 & 38,5 \\
\hline De pie & 213 & 55,8 \\
\hline Sentada & 22 & 5,8 \\
\hline ¿En qué posición palpa sus senos? & Frecuencia absoluta & Frecuencia relativa \\
\hline Acostada & 129 & 33,8 \\
\hline De pie & 217 & 56,8 \\
\hline Sentada & 27 & 7,1 \\
\hline Acostada y de pie & 9 & 2,4 \\
\hline ¿Con que parte de la mano palpa sus senos? & Frecuencia absoluta & Frecuencia relativa \\
\hline Los dedos & 39 & 10,2 \\
\hline Toda la mano & 23 & 6,0 \\
\hline Yema de los dedos & 320 & 83,8 \\
\hline ¿Cómo examina los senos? & Frecuencia absoluta & Frecuencia relativa \\
\hline Ambos la vez & 47 & 12,3 \\
\hline Uno la vez & 335 & 87,7 \\
\hline ¿Cuándo examina sus senos, incluye el examen del pezón? & Frecuencia absoluta & Frecuencia relativa \\
\hline NO & 74 & 19,4 \\
\hline SI & 308 & 80,6 \\
\hline
\end{tabular}


y otro tipo de información, que permita enriquecer el análisis estadístico de los resultados.

Conflicto de intereses: Este manuscrito fue elaborado y revisado con la participación de todos los autores, quienes declaramos no tener conflicto de intereses que ponga en riesgo la validez de los resultados.

\section{BIBLIOGRAFÍA}

1. ARCE, C.; BARGALLÓ, E.; VILLASEÑOR, Y.; GAMBOA, C.; LARA, F.; PÉREZ, V.; VILLARREAL, P. 2011. Onco guía cáncer de mama. Rev. Inst. Nal. Cancerol. Méx. 6:77-86.

2. BELL, H.; ODUARDO, O. 2015. Autoexamen de mama en mujeres de la Comunidad «19 de Abril» del municipio venezolano de Guanare.Rev. MEDISAN.19 (2):186-191.

3. CERVANTES, A.; DÍAZ, L.; SUDEA, M. 2011. Conocimientos y prácticas del autoexamen de mama como medida de detección temprana para el cáncer de mama en mujeres que asisten al CAP de la esperanza. Cartagena de Indias durante el primer trimestre del año 2011. Colombia. Disponible desde Internet en: http://siacurn.curnvirtual.edu.co:8080/ xmlui/bitstream/handle/123456789/571/CONOCIMIENTO\%20Y\%20PR\%c3\%81CTICAS\%20DEL\%20 AUTOEXAMEN\%20DE\%20MAMA.pdf?sequence $=1$ (con acceso 08/08/2015).

4. CRUZ, R.; CABALLERO, D. 2011. Problemas sociales relacionados con el estado de salud periodontal de la población infantil urbano-marginal venezolana. Rev. Méd. Electrón. 33(5):656-664.

5. DADIS. 2013. Análisis de la mortalidad por neoplasias en el distrito de Cartagena. Disponible desde Internet en: http://www.dadiscartagena.gov.co/images/docs/ saludpublica/vigilancia/informe_de_mortalidad_neoplasias_2013.pdf (con acceso 9/09/15).

6. DEPARTAMENTO ADMINISTRATIVO NACIONAL DE ESTADÍSTICA DANE. 2010. Estimaciones de población 1985-2005 y proyecciones de población 2005-2020 nacional y departamental desagregado por área, sexo y grupos quinquenales de edad. Disponible desde internet en: http://www.dane.gov.co/ index.php/poblacion-y-demografia/proyecciones-depoblacion (con acceso 05/08/2015).

7. ENCUESTA NACIONAL DE DEMOGRAFÍA Y SALUD -ENDS-. 2010. Detección temprana de Cáncer de mama. Disponible desde Internet en https://dhsprogram.com/pubs/pdf/FR246/FR246.pdf (con acceso 25/04/13).

8. FERLAY, J.; STELIAROVA-FOUCHER, E.; LORTETTIEULENT, J.; ROSSO, S.; COEBERGH, J.W.; COMBER, H.; FORMAN, D.; BRAY, F. 2013.Cancer incidence and mortality patterns in Europe: estimates for 40 countries in 2012. Eur. J. Cancer. 49(6):13741403.

9. FERNÁNDEZ, L.; SÁNCHEZ, Y.; PIÑA, D.; DE LA PAZ, K. 2012. Modificación de conocimientos sobre el autoexamen de mamas en mujeres de la Policlínica Universitaria "René Vallejo Ortiz". Rev. MEDISAN.16 (11):17-24.

10. GUTIÉRREZ DELGADILLO, A.; GUTIÉRREZ FLORES, F.R.; GUTIÉRREZ CHOQUE, J.C.; GUZMÁN DELGADILLO, F.; SANTANDER LÓPEZ, A. 2012. Conocimiento, actitud y práctica del autoexamen mamario en mujeres estudiantes de medicina de la Universidad Mayor de San Simón Gestión 2011. Rev. Cient. Cienc. Méd. 15(2):22-25.

11. MARTÍNEZ, L.; CRUZ, L.; LÓPEZ, M.; CRUZ, C.; MUIÑO, M. 2012. Valoración del conocimiento del autoexamen de mama en mujeres en edad reproductiva del Policlínico Bernardo Posse. Rev Habanera Cienc. Médicas. 11(3):361-368.

12. MENDOZA, B. 2013. Cultura de prevención sobre autoexamen de mama y estudio mamográfico en pacientes atendidas en el servicio de mamografía del hospital nacional Edgardo RebagliatiMartins - EsSalud. Disponible desde Internet en: http://cybertesis. unmsm.edu.pe/handle/cybertesis/3475 (con acceso 6/08/15).

13. MINISTERIO DE SALUD. 2000. Norma técnica para la detección temprana del cáncer de seno. Disponible en Internet en: http://odm.colnodo.apc.org/apc-aa-fi les/5774d034c9be96efe0bff91311203bc4/Norma tecnica_para_la_deteccion_temprana_del_cancer_ de_seno.pdf (con acceso 10/08/15).

14. MINISTERIO DE SALUD Y PROTECCIÓN SOCIAL. 2014. Cáncer de mama, una enfermedad en ascenso en Colombia. Disponible desde Internet en: https://www.minsalud.gov.co/Paginas/-Cancer-demama,-una-enfermedad-en-ascenso-en-Colombia. aspx (con acceso 01/12/15). 
15. OCHOA, F.; VEGA, B.; ARÉVALO, P. 2015. Conocimientos, actitudes y prácticas del autoexamen de mamas en mujeres del área rural. Cuenca, 2014. Disponible desde Internet en:http://dspace.ucuenca.edu.ec/ handle/123456789/21207 (con acceso 05/08/15).

16. OMS. 2009. Cáncer de mama: prevención y control. Disponible desde Internet en: http://www.who.int/topics/ cancer/breastcancer/es/(con acceso 06/08/15).

17. OSPINA, J.; MANRIQUE, F.; VEGA, N.; MORALES, A.; ARIZA, R. 2011. La no realización del autoexamen de mama en Tunja, Colombia. Rev. Enferm Global 10(3):30-40.

18. PARDO, S. 2011. Modificación de conocimientos sobre cáncer de mama en trabajadoras con factores de riesgo de la enfermedad. Rev.MEDISAN.15 (1):1-7.

19. SÁENZ, A.; SÁNCHEZ, A. 2010. Conocimientos, actitudes y prácticas del autoexamen mama en mujeres de 15 - 45 años que acuden a los consultorios de Planificación Familiar y Papanicolaou del Hospital Nacional Daniel Alcides Carrión, periodo agosto diciembre del 2010. Disponible desde Internet en: http://cybertesis.unmsm.edu.pe/handle/cybertesis/3012 (con acceso 05/03/13).

20. SILVA, N.; ARRUDA, D.; REGO, S. 2013.Conocimiento y práctica del autoexamen de mamas por usuarias de la Unidad de Salud de la Familia. Enferm. Glob. 12(29):463-476.

21. SUASNABAR, S.; RAMÍREZ, E. 2011. Relación entre el nivel de conocimientos y las prácticas sobre la prevención del cáncer de mama en las mujeres que acuden al C.S. Villa San Luis: San Juan de Miraflores. Disponible desde Internet en:http://cybertesis. unmsm.edu.pe/xmlui/bitstream/handle/cybertesis/1309/Suasnabar_rs.pdf?sequence $=1$ (con acceso $02 / 12 / 15)$.

22. TORO, M.; PEINADO, L.; DÍAZ, A. 2014. Conocimientos y prácticas sobre el auto examen mamario en mujeres de Cartagena de Indias (Bol. -Col). Rev. Cienc. Innov. Salud. 2(1):27-32.

23. VIDAL, D.; CHAMBLAS, I.; ZAVALA, M.; MÜLLER, R.; RODRÍGUEZ, M.; CHÁVEZ, A. 2014. Determinantes sociales en salud y estilos de vida en población adulta de Concepción. Rev. Cienc. Enferm. 20(1):61-74.

24. YÉPEZ, D.; DE LA ROSA, A.; GUERRERO, C.; GÓMEZ, M. 2012. Autoexploración mamaria: conocimiento y perspectiva en mujeres. Rev. Enferm. Inst. Mex.SeguroSoc.20 (2):79-84.

Recibido: Diciembre 24 de 2015

Aceptado: Abril 18 de 2016

Cómo citar:

Castillo, I.; Bohórquez, C.; Palomino, J.; Elles, L.; Montero, L. 2016. Conocimientos, actitudes y prácticas sobre el autoexamen de mama en mujeres del área rural de Cartagena. Rev. U.D.C.A Act. \& Div. Cient. 19(1):5-14. 